\title{
Obesity and weight control: the evidence
}

\author{
Jacob C. Seidell \\ National Institute of Public Health and the Environment, Bilthoven, The Netherlands
}

The evidence will be discussed at four levels.

Associations have been established between measures of obesity and cardiovascular disease risk factors. Crude measures of body fatness such as BMI or waist circumference are both positively related to blood pressure, serum triacylglycerols, small dense LDL-cholesterol, glucose and insulin concentrations, and haemostatic factors and fibrinolysis. There is a negative association between measures of obesity and HDL-cholesterol.

In prospective studies high levels of BMI and waist are predictive of future cardiovascular disease. The relative and absolute risks of obesity are dependent on sex and age of subjects as well as the length of follow up as well as type of end points. For CHD the relative risk for individuals with BMI $>30 \mathrm{~kg} / \mathrm{m}^{2}$ is about 2-3 compared with those with a BMI $<25 \mathrm{~kg} / \mathrm{m}^{2}$. The risks for stroke have been less consistent and seem to be less high than expected on the basis of the relationship between BMI and blood pressure. Observational studies show that weight gain during adult life is, independently of the extent of obesity, associated with increased risk of cardiovascular disease. Weight loss has been found to predict increased risk of CHD in observational studies, but distinction between effects of intentional and unintentional weight loss is usually not possible.

Randomized clinical trials on weight loss and cardiovascular disease risk factors show that the effects depend on the mode of weight loss (by dietary modification and/or physical activity or pharmacotherapy), the extent of weight loss, and characteristics of the subjects studied (e.g. hypertensive subjects $v$. non-hypertensive subjects, diabetic subjects $v$. non-diabetic subjects etc.). The National Institutes of Health, National Heart, Lung and Blood Institute (1998) has critically evaluated the available literature. The report concluded that weight loss by dietary restriction is recommended to lower increased blood pressure, elevated levels of total cholesterol, LDL-cholesterol and triacylglycerols, and to raise low levels of HDL-cholesterol and to lower elevated glucose levels in overweight and obese subjects with type 2 diabetes. There are also favourable effects on haemostasis, fibrinolysis and heart function. It is not always clear which effects can be ascribed to weight loss per se and which to the dietary modification itself (Seidell, 1999).

Randomized clinical trials have been conducted on the effects of weight loss on subsequent incidence of cardiovascular disease. There are no randomized clinical trials that have allowed the study of cardiovascular end points. The only study which will be able to study the effects of weight loss on cardiovascular disease in the near future is the semi-randomized Swedish Obese Subjects trial which is a large national surgical intervention trial in morbidly-obese subjects (Sjöström et al. 1999). The 4-year results have shown that massive weight loss is favourably affecting risks for cardiovascular disease, but full analyses have not been released. Given the poor success rate of long-term weight loss by lifestyle modification and pharmacotherapy it is unlikely that major trials will be performed or will have sufficient power to detect effects of weight loss on the incidence of CHD and stroke.

\section{Conclusions}

Circumstantial evidence suggests that maintained weight loss will produce a reduction in CVD risk. Long-term effects of weight loss, however, are not available and the unexpected increased risk of CHD in some observational studies cannot be dismissed lightly. Long-term randomized clinical trials should be encouraged to quantify the effectiveness of weight loss on specific end points.

\section{Cardiovascular disease: Risk factors: Obesity: Weight loss}




\section{References}

National Institutes of Health, National Heart, Lung and Blood Institute (1998) Clinical guidelines on the identification, evaluation, and treatment of overweight and obesity in adults the evidence report. Obesity Research 6, Suppl. 2, 51S-209S.
Seidell JC (1999) Optimizing fat intake: does a reduction in fat intake prevent obesity? European Heart Journal 1, Suppl. S, S118-S122.

Sjöström CD, Lissner L, Wedel H \& Sjöström L (1999) Reduction in incidence of diabetes, hypertension and lipid disturbances after intentional weight loss induced by bariatric surgery: the SOS intervention study. Obesity 7, 477-484.

(C) Nutrition Society 2000 